\title{
WHAT IS THE VALUE OF APPLYING SOCIAL THEORY TO MATERNITY CARE?
}

Pregnancy, birth and transition to motherhood are physiological processes, but they are always socially and culturally shaped and managed, forming a major event in the lives of individual women, families and communities. Childbirth is not only about individual reproduction - having a child, becoming a mother - but about social reproduction - the way in which her society and culture is reproduced. As a result, and because humans are essentially social beings, childbirth is always socially and culturally shaped and managed. In a parallel way, the roles and professional status of midwives, obstetricians and nurses are shaped by the social and cultural contexts in which they work to support women in pregnancy, birth and the transition to parenthood.

Giving birth is a process which creates new social persons and roles: the foetus becomes a social person and the woman giving birth becomes a mother, her partner a father and new families are formed. As a consequence, the ways in which women give birth are a matter of very wide interest - an object of policy and a subject of social interest and concern. The ways in which childbirth is shaped and managed by legal and government frameworks, by policies, by professionals and institutions is reflective of the social and cultural context, and shaped by its history. We can borrow the anthropologist Mary Douglas's concept - the body as a microcosm - to talk about how the social world is inscribed on women's bodies and about how women's bodies and reproduction represent that social world. The idea of the body as a microcosm provides a picture at the micro-level of the body of structures and relationships at the macro-level of its social context. We can talk also of how the process of birth and the ways in which it is managed is not only influenced by the social world but also, in turn, helps to shape the social world of which it is a part.

I hope by this introduction to have shown why social theory should be useful in thinking about childbirth and maternity care. I now want to suggest some ways in which social theory can form an important tool for critical thinking and analysis, which helps nurses and midwives to foster the skills and knowledge to develop, interpret, question, debate and apply evidence in practice.

At the level of public health (at the macro-level of analysis), social theory helps to analyse and explain the social determinants of health and the complexity of the ways in which socio-economic conditions, life chances, access to knowledge and power can shape health status and wellbeing. Maternity care is central to public health as wellbeing in pregnancy and the perinatal period profoundly influences the future health of the child and even following generations as well as the health of the mother. The impact of healthcare, even maternity care, takes place at a less fundamental level. At the outset of pregnancy, the health of some women and their babies is already disadvantaged through social inequalities based on class, ethnic background and residence. Inequalities are also gendered, as is the power to influence social structure and policy, the delivery of healthcare and relationships in care. So, even before the journey of pregnancy and birth begins, social theory should play a role in informing maternity professionals about the factors influencing maternal and infant health. Such factors have also been shown to influence both the access to care and the quality of care received by different women in society, in such a way that maternity care may compound, rather than counter, existing social inequalities in health. Political economy can help to explain both the inequalities in the care received by different women, but also the inequalities of power between obstetricians, nurses and midwives and the economic and institutional drivers for medicalization of care.

If we turn to the healthcare institutions such as hospitals and clinics and the professions such as obstetrician or midwife (the meso-level of analysis), we can use social theory in order to analyse and understand the ways in which care is organised and provided, the division of professional roles and the attitudes and practices of those professionals, as well as their inter-relationships. Theories of gender, for example, can help to explain how the autonomy of midwives and their scope of practice have been constrained, and how obstetric knowledge came to be seen as more authoritative, while theories of medicalization and of modernity can illuminate the reasons for the excessive and socially unequal ways in which technology is applied in childbirth. 
One example from my own research work has been in using social theory to analyse the ways in which time is conceptualised and managed in childbirth and maternity care. I noticed that historically, the concepts of duration of labour changed and an emphasis on measurement and control of time in pregnancy and birth developed. This shift in maternity care traced larger historical shifts in cultural concepts of time and in how we manage and think about time in our everyday and working lives. It is reflected in the emphasis in managing time in pregnancy and birth, but similarly in the risk of surveillance technology, risk-management and of protocol-based care. My work in evaluating the 'caseload' model of midwifery, in which midwives cared for a defined caseload of women, maintaining a high level of continuity, autonomy of practice and accountability for their care highlighted how the management and even their concepts of time in childbirth began to shift as these midwives adapted to their 'new' role, which was more akin to the role of the traditional midwife, or parteira. These midwives seemed to operate with a post-modern concept of time, however - a concept which was more fluid and woman-centred. It had much in common with the time concepts and practices of more traditional societies and traditional midwives, and with a focus on active physiological birth rather than 'active management of' birth, but within a late modern context of establishing their status as a professional group, of using scientific evidence critically and asserting their professional autonomy and responsibility.

Several of the articles in this journal issue reflect the importance social theories, such as those of kinship in nursing research, in terms of the roles of family members, the nature of the family and how being a mother or other family member is conceptualised and experienced in relation to illness, disability or frailty. Being the parent of a child with a disability, for example, challenges common assumptions about motherhood in terms of the maternal role and how this changes over time with the child's development. It invites consideration of the experience of motherhood and the social recognition of the family roles in a context of social stigma. The articles in this issue also reflect the ways in which social forces shape the practices and self-concepts of nurses and those of the general population, in ways that impact on health and care. They also demonstrate ways in which the subjective experiences of providing or receiving healthcare may impact on the outcomes of that care.

Christine McCourt

Ph.D. Professor of Maternal and Child Health, School of Health Sciences, City University London 